\title{
The universal equation of state of a unitary fermionic gas
}

\author{
R.K. Bhaduri, ${ }^{1}$ W. van Dijk, ${ }^{1,2}$ and M. V. N. Murthy ${ }^{3}$ \\ ${ }^{1}$ Department of Physics and Astronomy, McMaster University, Hamilton, Ontario L8S 4M1, Canada \\ ${ }^{2}$ Physics Department, Redeemer University College, Ancaster, Ontario L9K 1J4, Canada \\ ${ }^{3}$ The Institute of Mathematical Sciences, Chennai 600113, India
}

(Dated: August 9, 2018)

\begin{abstract}
It is suggested that for a fermi gas at unitarity, the two-body bond plays a special role. We propose an equation of state using an ansatz relating the interaction part of the $l$-body cluster to its two-body counterpart. This allows a parameter-free comparison with the recently measured equation of state by the ENS group. The agreement between the two over a range of fugacity ( $z<5$ for a homogeneous gas, and $z<10$ for the trapped gas) leads us to perform the calculations of more sensitive quantities measured recently by the MIT group.
\end{abstract}

Feshbach resonance makes it possible to adjust the strength of the inter-atomic interaction in a neutral atomic gas. When the scattering length goes to $\pm \infty$, there is no length scale left other than the average inter-particle distance and the thermal wavelength (assuming a zero-range interaction). The gas is then termed "unitary" and its properties are universal when expressed in appropriate dimensionless units at all scales whether the system is fermionic or bosonic [1]. Recent accurate measurements by the ENS group [2, 3] and the Tokyo group [4] have confirmed the universal nature of the equation of state (EOS) of a gas of neutral fermionic atoms, and have given fresh impetus to its theoretical understanding [5, 6]. More recently, direct measurements by the MIT group [7] of the isothermal compressibility $\kappa$, pressure $P$, and heat capacity $C_{V} / N k_{B}$ for a unitary gas have revealed the superfluid transition at $T_{c} / T_{F}=0.167(13)$.

In this paper, keeping in mind the fundamental nature of the two-particle bond at unitarity, we propose a description of the unitary gas as consisting of singlet pairs, in terms of which all higher order clusters are expressed. The resulting Equation of State (EOS) extends the agreement with the ENS data [2, 3] on the grand potential over a much larger range of fugacity $z$ than expected. However, this description breaks down for $z>5$ for the homogeneous gas (and $z>10$ for the harmonically trapped gas). For the homogeneous gas, $z=5$ corresponds to a temperature $T / T_{F}=0.22$, below which the proposed EOS cannot be trusted. We calculate, with our higher virial coefficients, the pressure, compressibility and heat capacity of the homogeneous gas to compare with the MIT data [5]. The calculation of these quantities is a stringent test since they require higher moments of the virial expansion. We find that inclusion of the higher virial coefficients yields agreement with the MIT data for pressure and entropy down to $T / T_{F}=0.3$, and the compressibility and heat capacity to $T / T_{F}=0.6$.

To set the stage for the proposed universal EOS, we briefly recapitulate the virial expansion of a two-component interacting homogeneous fermi gas [8]. The grand potential $\Omega(\beta, \mu)$ is defined as $\Omega=-\tau \ln \mathcal{Z}$, where $\tau=k_{B} T=1 / \beta$ and $\mathcal{Z}$ is the grand-canonical partition function. Furthermore $\Omega=-P V$, and may be expressed in a power series of fugacity $z=\exp (\beta \mu)$, where $\mu$ is chemical potential. The grand potential $\Omega=-\tau Z_{1}(\beta) \sum_{l=1}^{\infty} b_{l} z^{l}$, where $Z_{1}(\beta)$ is the one-body partition function, and $b_{l}$ is the $l$-particle clus- ter integral. For an untrapped gas in volume $V$, we have $Z_{1}(\beta)=2\left(V / \lambda^{3}\right)$, where spin degeneracy of 2 is included and $\lambda=\left(2 \pi \hbar^{2} \beta / m\right)^{1 / 2}$ is the thermal wave length. For a harmonic oscillator (HO) trap in three dimensions, $Z_{1}(\beta)=$ $2 /(\hbar \omega \beta)^{3}$. For a unitary gas, the cluster integrals $b_{l}$ 's are also temperature independent in the high-temperature expansion. Subtracting from $\Omega$ the ideal part of the grand potential $\Omega^{(0)}$ we obtain the interaction part of the EOS as

$$
\Omega-\Omega^{(0)}=-\tau Z_{1}(\beta) \sum_{l=2}^{\infty}\left(\Delta b_{l}\right) z^{l},
$$

where $\Delta b_{l}=b_{l}-b_{l}^{(0)}$. Note that $b_{1}=b_{1}^{(0)}=1$, and cancels out on taking the difference.

Consider now the special role played by $\Delta b_{2}$ of the twoparticle cluster at unitarity. In such a gas, the spin-up fermions have a tendency to pair up with the spin-down fermions because the short-range interaction potential is on the verge of producing zero-energy bound states. The Feshbach resonance being in the relative $s$-state, ensures the pair interaction to be operative only between singlet pairs. One finds that [9] $\Delta b_{2}=(2 \sqrt{2}) \times \frac{1}{2}\left(\Delta Z_{2}\right)$, where the factor $2 \sqrt{2}$ arises from the CM motion, $\Delta Z_{2}$ is the relative two-body partition function, and the "suppression factor" $\frac{1}{2}$ arises from the fact that only half of the $N$ particles can interact in a spinbalanced two-component Fermi gas. Note that [10] at unitarity $\Delta Z_{2}=\frac{1}{2}$, yielding $\Delta b_{2}=\frac{1}{\sqrt{2}}$ for such a system. What about the $\Delta b_{l}$ 's for the $l$-body clusters that appear in Eq. (1)? Keeping in mind that the unitary gas may be looked upon as a system consisting of forming and dissolving two-body pairs, we conjecture that for the scale invariant system, the $\Delta b_{l}$ for $l>2$ should be expressible in terms of $\left(\Delta b_{2}\right)$ with an appropriate suppression factor. Viewing a $l$-body cluster as one particle interacting with the rest from a cluster of $(l-1)$ paired particles, we assume that the suppression factor is given by $2^{\mathcal{N}_{(l-1)}}$, where $\mathcal{N}_{(l-1)}=(l-1)(l-2) / 2$ is in general the number of pairs in a cluster with $(l-1)$ fermions. Thus our basic ansatz is

$$
\Delta b_{l}=(-)^{l} \frac{\left(\Delta b_{2}\right)}{2^{\mathcal{N}_{(l-1)}}}, l \geq 2 .
$$

For $l=2, \mathcal{N}_{1}=0$, and Eq. (2) is an identity. The alternating sign $(-)^{l}$ in the above equation was put in to keep the number fluctuation $(\Delta N)^{2} / \bar{N}=\sum_{l} l^{2} b_{l} z^{l} / \sum_{l} l b_{l} z^{l}$ not to grow 
to a very large value with $z$, where $(\Delta N)^{2}=\overline{N^{2}}-\bar{N}^{2}$ is the number fluctuation, proportional to the isothermal compressibility [11]. A large value of the compressibility would lead to a vanishing monopole excitation which is a signature of instability [12].

Although our description of the higher virial coefficients in terms of the second may seem to be very different from the conventional one, similar relationship between the third and second virial coefficients have been found in anyons which is also a scale invariant system [13, 14]. This is obtained by demanding that the divergences in the three-body clusters cancel by similar divergences in two-body clusters in the high temperature limit. A formal derivation for arbitrary $l$ for the unitary gas appears to be non-trivial.

With this ansatz,

$$
\Omega-\Omega^{(0)}=-\tau Z_{1}(\beta)\left(\Delta b_{2}\right) \sum_{l=2}^{\infty}(-)^{l} \frac{z^{l}}{2^{\mathcal{N}_{(l-1)}}} .
$$

Experimentally [2, 5, 15], it is the quantity $h(\zeta)=\Omega / \Omega^{(0)}$ that is extracted, where $\zeta=1 / z$. This is given by

$$
h(\zeta)=1+\left(\Delta b_{2}\right) \frac{\sum_{l=2}^{\infty}(-)^{l}(\zeta)^{-l} / 2^{\mathcal{N}_{(l-1)}}}{\tilde{\Omega}^{(0)}} .
$$

In a homogeneous gas with a spin-degeneracy of 2,

$$
\tilde{\Omega}^{(0)}=\frac{2}{\sqrt{\pi}} \int_{0}^{\infty} \sqrt{t} \ln \left(1+z e^{-t}\right) d t
$$

It is worth noting that using Eq. (2) with $\Delta b_{2}=1 / \sqrt{2}$, we obtain $\Delta b_{3}=-1 / 2 \sqrt{2}, \Delta b_{4}=1 / 8 \sqrt{2}, \Delta b_{5}=$ $-1 / 64 \sqrt{2}$, etc. Numerically $\Delta b_{3}$ is known to great accuracy, it was calculated up to 8 decimal figures in [16] and has now been improved to 12 decimal figures [17]. Our ansatz for the third virial coefficient differs from the numerically computed value in the third decimal and as such cannot be exact. However, as we shall see the agreement with EOS data is unaffected by such fine differences in $\Delta b_{3}$. It is also estimated [2] that $\Delta b_{4} \approx 0.096 \pm 0.015$, and is consistent with our prediction within the error bars. It should be mentioned that $\Delta b_{4}$ as quoted in [17] is different sign and magnitude from [2] and our value. This however destroys the agreement with the data from the ENS group.

Before confronting the experimental data, we note that for a gas trapped in a three-dimensional harmonic oscillator (HO), Eqs. (4) is modified to [2, 5]

$$
h(\zeta)=1+\left(\Delta b_{2}\right) \sum_{l=2}^{\infty} \frac{(-)^{l}}{(l)^{3 / 2}} \frac{\zeta^{-l}}{2^{\mathcal{N}_{(l-1)}}} / \widetilde{\Omega}^{(0)},
$$

and $\widetilde{\Omega}^{(0)}=\frac{1}{2} \int_{0}^{\infty} t^{2} \ln \left(1+z e^{-t}\right) d t$. The additional suppression factor of $1 / l^{3 / 2}$ in Eq. [5] was derived in [16] assuming a local fugacity in a $\mathrm{HO}$ potential.

We are now ready to compare our predictions given by Eq. (4) for the homogeneous gas and Eq. (5) for the HO with experimental data. In Fig. 1 $h(\zeta)-1$ for the homogeneous unpolarized gas, as given by our Eq. (4), is plotted against

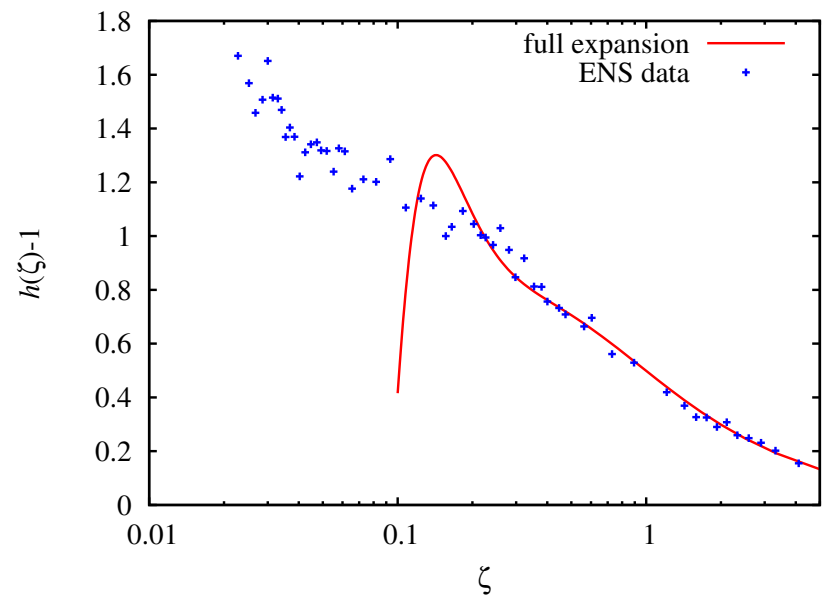

FIG. 1. (Colour online) The function $h(\zeta)-1$ for the untrapped unitary Fermi gas (from Eq. (4) as a function of $\zeta$. The crosses represent the experimental data presented by Nascimbène et al. [2] in their Fig. 3a.

$\zeta$. The crosses on the plot are the ENS experimental data as found by Nascimbéne et al. [2] The authors quote that $h$ and $\zeta$ are accurate to within 6 percent. It will be seen from this figure that the series given by our Eq. (4) is in good agreement with the data down to $\zeta \approx 0.2$. To put the agreement in perspective the same data are plotted as a function of $z=1 / \zeta$ in Fig. 2. along with the behavior of the EOS including virial co-

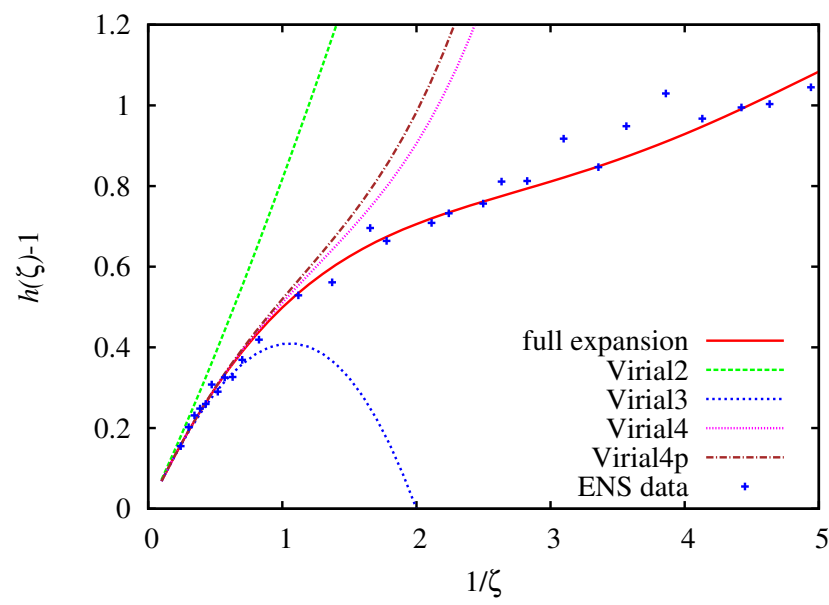

FIG. 2. (Colour online) The universal function $h(\zeta)-1$ for the the untrapped fermi gas plotted as a function of the fugacity $1 / \zeta$. The 2nd, 3rd, and 4th virial expansions are also shown. The 4th order expansion labelled Virial4p has $\Delta b_{4}=0.096$. The experimental data are the same as in Fig. 1

efficients up to the fourth order, as was done by $\mathrm{Hu}$ et al. [5] We see that such a truncated series could match the data to about $z \approx 1.7$. Our series (4) extends this to about $z \approx 5$. This also underlines the importance of higher-order virial coefficients $\Delta b_{l}$ 's for $l>4$, despite their rapidly diminishing values. For the curve labelled Virial4p we set $\Delta b_{4}=0.096$, the estimated value [2], rather than 0.088 given by our ansatz. 
Note that the $h(\zeta)$ has been calculated in [6] within Pade approximation and including up to $\Delta b_{3}$. Despite deviation from ENS data for $\zeta<1$, they obtain surprisingly good agreement for energy and entropy per particle down to $T / T_{F}=0.16$.

We now turn to the ENS measurement for the trapped unitary unpolarized gas, as extracted by $\mathrm{Hu}$ et al. [5], and compare with our Eq. (5). (See Fig. 3) ) Here the convergence of

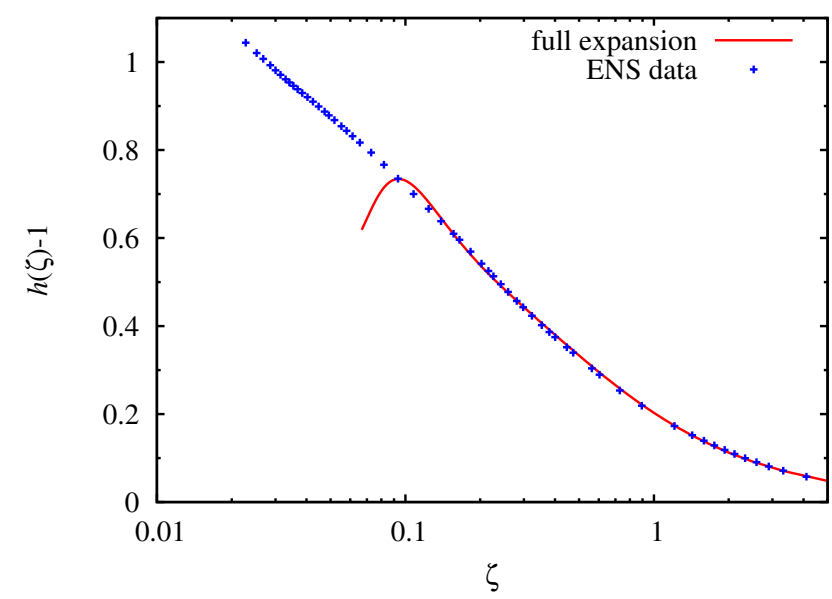

FIG. 3. (Colour online) The universal function $h(\zeta)-1$ for fermions in an harmonic trap as a function of $\zeta$, Eq. 5]. The crosses represented the experimental data presented in Fig. 6 of Hu et al. [5]

the virial series is faster as expected, and the agreement is remarkably good down to $\zeta \approx 0.1$. Figure 4 shows this clearly

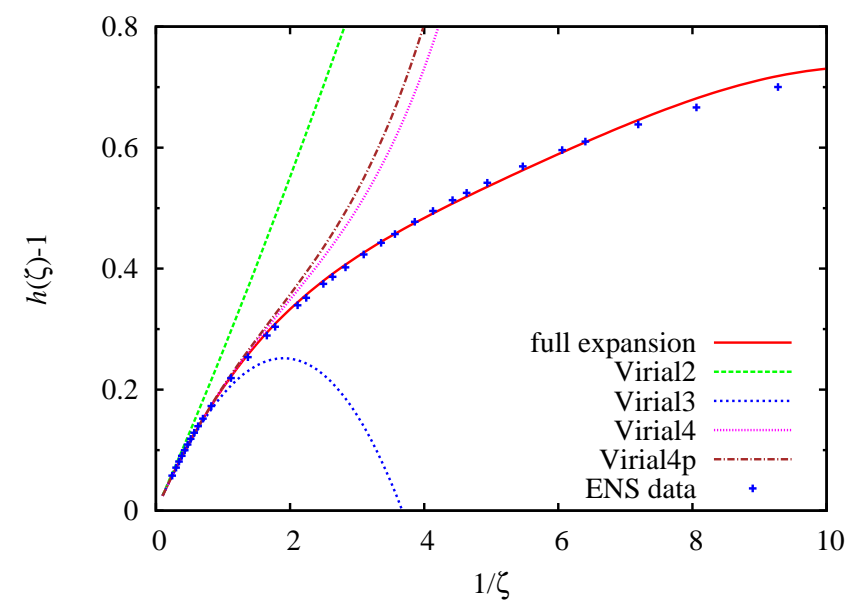

FIG. 4. (Colour online) The universal function of trapped fermions $h(\zeta)-1$ as a function of fugacity. See Eq. [5]. Virial4p is the 4thorder virial expansion with $\Delta b_{4}=0.096$. The experimental data are the same as in Fig. 3

when the truncated predictions from previous work are compared with our result. The range of applicability of the virial series (5) is now extended fourfold to $z \approx 10$.

It should be noted that the series given in Eqs. (4) and (5) converge for any value of $z$. However, the range of validity of the sum depends on the maximum value of $l$ as seen from Fig. 2 and Fig. 4 for free gas and HO respectively. The first few terms make a significant difference but the importance of $\Delta b_{l}$ for $l>8$ is minimal even at $z=5$. The series gets saturated by the first twenty terms which is denoted as "full expansion" in all the figures.

Encouraged by the agreement with $h(z)$ even deep into region $z>1$ where the normal virial expansion is not expected to work, we next compare our predictions for the recently measured data on compressibility, heat capacity, and pressure by the MIT group [7]. Following their notation we write $\beta P=f_{P}(X) / \lambda^{3}$, where $P$ is the pressure, $X=\ln z=\beta \mu$ and $f_{P}(X)$ is the universal function given by

$$
f_{P}(X)=2\left(f_{5 / 2}(\exp (X))+\sum_{l=2}^{\infty} \Delta b_{l} \exp (l X)\right) .
$$

The first term in the bracket is the contribution due to the ideal fermi gas, and is the standard fermi-dirac integral [18]. All thermodynamic quantities can now be expressed in terms of this universal function and its derivatives. Specifically we have pressure and compressibility normalized by their zero temperature values, $P_{0}, \kappa_{0}$, given by

$$
\tilde{p}=\frac{P}{P_{0}}=\frac{5 T}{2 T_{F}} \frac{f_{P}(X)}{f_{P}^{\prime}(X)} ; \quad \tilde{\kappa}=\frac{\kappa}{\kappa_{0}}=\frac{2 T_{F}}{3 T} \frac{f_{P}^{\prime \prime}(X)}{f_{P}^{\prime}(X)},
$$

where $T / T_{F}=4 \pi /\left[3 \pi^{2} f_{P}^{\prime}(X)\right]^{2 / 3}$ is the dimensionless temperature scale and the prime denotes a derivative with respect to $X$. The heat capacity at constant volume and entropy are given by $C_{V} / N k_{B}=\frac{15}{4} \frac{f_{P}(X)}{f_{P}^{\prime}(X)}-\frac{9}{4} \frac{f_{P}^{\prime}(X)}{f_{P}^{\prime \prime}(X)}=$ $\frac{3 T_{F}}{2 T}(\tilde{p}-1 / \tilde{\kappa}), S / N k_{B}=\frac{5}{2} \frac{f_{P}(X)}{f_{P}^{\prime}(X)}-\ln (z)$. The above expressions allow one to calculate the relevant quantities either as a function of fugacity $z$ or temperature $T / T_{F}$ using the virial expansion given by Eq. (6) and compare with the respective experimental data of $\mathrm{Ku}$ et al.

In the light of our earlier remarks (see Fig. 1), these comparisons are limited to $z$ values less than 4.95 , corresponding to $T / T_{F}>0.22$. Fig. 5 shows the variation of the pressure, entropy, and heat capacity as a function of $T / T_{F}$. The agreement with experimental data improves noticeably as the higher $\Delta b_{l}$ 's are included. The agreement for the pressure and entropy hold to $T / T_{T}=0.3$, indicating that the first moments of the virial expansion are good.This is not the case for the second moments, however, as the plots for heat capacity vs $T / T_{F}$ shows. The theoretical plots start deviating appreciably from the data for $T / T_{F}<0.6$. The same behavior is seen in Fig. 6 where the compressibility is plotted as a function of pressure (in reduced variables). It is interesting to note that despite these deviations, a peak in the compressibility of about the right magnitude appears in the theoretical curve, though at a higher value of $P / P_{0}$ or $T / T_{F}$. Though tempting, we are reluctant to interpret this as indicative of the onset of superfluidity in view of the inaccuracy of the virial description in this range of temperature or pressure.

We conclude that the high-temperature virial expansion, in conjunction with our ansatz, can match the EOS over a significantly larger range of fugacity, corresponding to about $T / T_{F} \approx 0.3$ for the homogeneous gas. Our ansatz (given by 

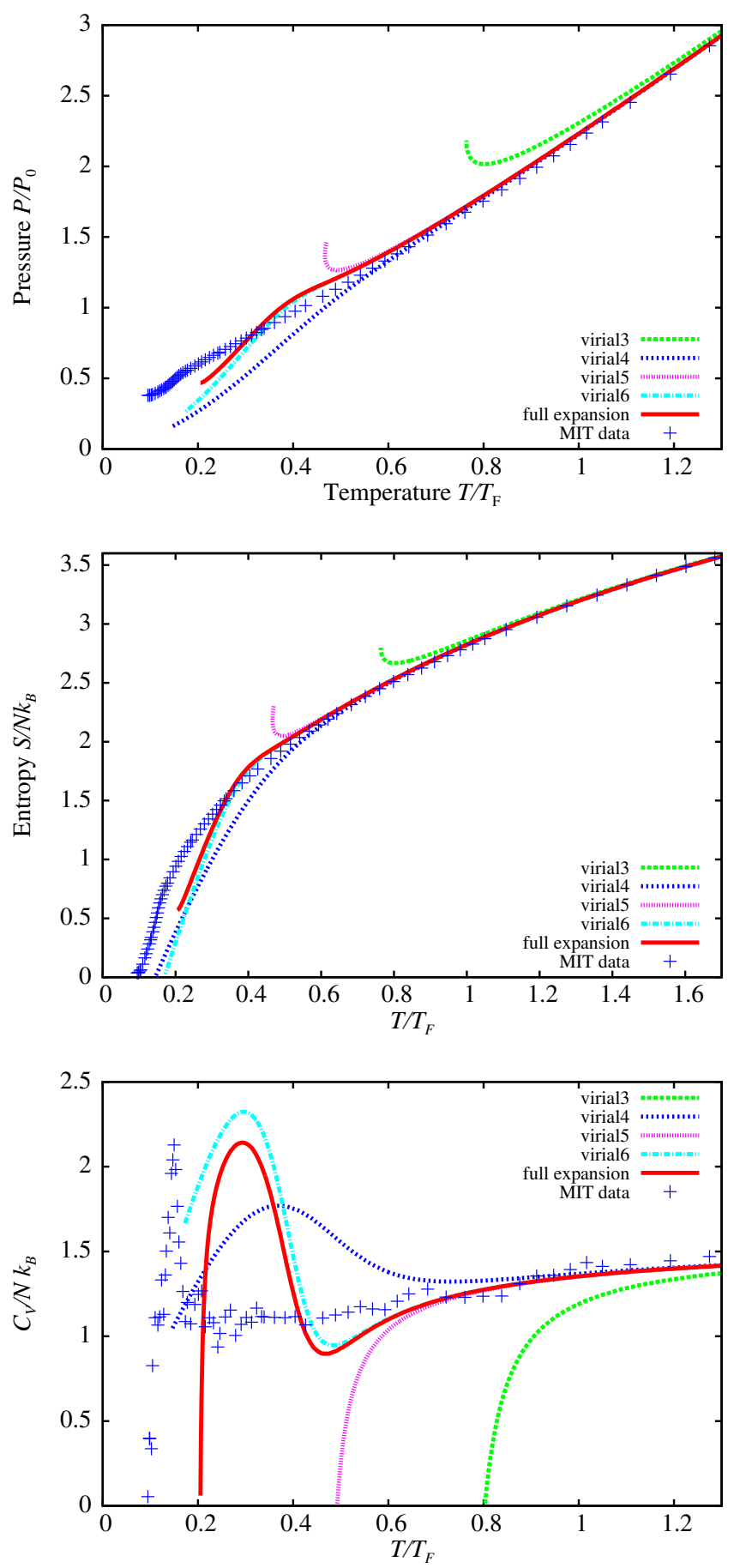

FIG. 5. (Colour online) Reduced pressure (top), entropy (middle) and heat capacity (bottom) shown as a function of $T / T_{F}$ for the untrapped unitary Fermi gas. The experimental data are taken from $\mathrm{Ku}$ et al. [7].

Eq. (2) resulted from the picture of a unitary fermi gas as a dynamic collection of singlet pairs, and assumed that $\left(\Delta b_{2}\right)$ determines the higher virial coefficients. The resulting success of this picture may point to some truth in this conjecture, and poses a challenge for deeper understanding.

We thank S. Nascimbène, H. Hu and M. Ku for sharing the

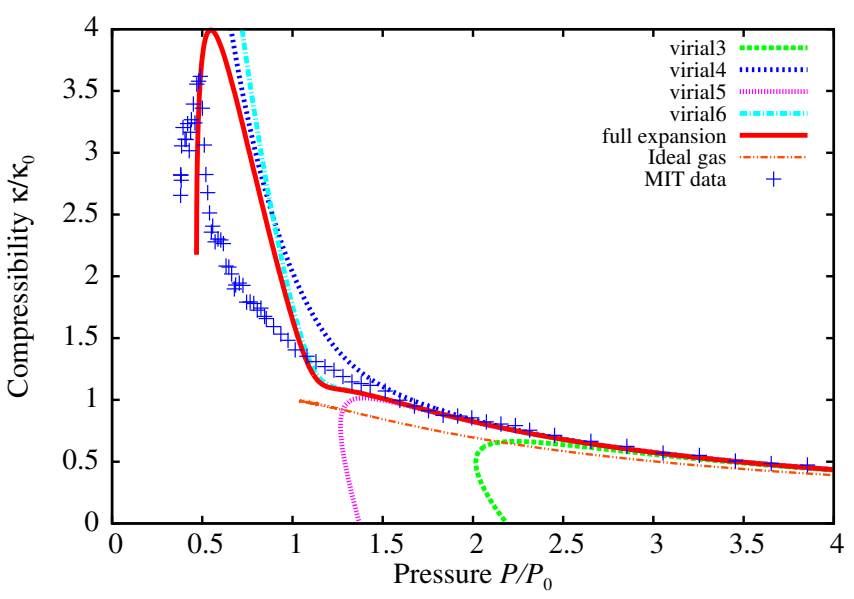

FIG. 6. (Colour online) Reduced compressibility shown as a function of reduced pressure for the untrapped unitary Fermi gas. The experimental data are taken from $\mathrm{Ku}$ et al. [7].

experimental data for the ideal and trapped fermion gas. We thank S. Das Gupta for helpful discussions. WvD acknowledges financial support from the Natural Sciences and Engineering Research Council of Canada, and MVN acknowledges the hospitality of Department of Physics and Astronomy, McMaster University where part of this work was done.

[1] T.-L. Ho, Phys. Rev. Lett. 92090402 (2004).

[2] S. Nascimbène, N. Navon, K.J. Jiang, F. Chevy, and C. Salomon, Nature 463, 1057 (2010).

[3] N. Navon, S. Nascibeène, F. Chevy, and C. Salomon, Science 328, 729 (2010).

[4] M. Horikoshi, S. Nakazima, M. Ueda, and T. Mukaiyama, Science 327, 442 (2010).

[5] H. Hu, X,-J. Liu, and P.D. Drummond, New Journal of Physics 12, 063038 (2010).

[6] H. Hu, X,-J. Liu, and P.D. Drummond, Phys. Rev.A 83, 063610 (2011).

[7] M.J.H. Ku, A.T. Sommer, L.W. Cheuk, and M. W. Zwierlein, Science 335, 563 (2012).

[8] T.-L. Ho and E.J. Mueller, Phys. Rev. Lett. 92, 160404 (2004).

[9] K. Huang, Statistical Mechanics (John Wiley, 1965) p. 309.

[10] E. Beth and G.E. Uhlenbeck, Physica IV, 915 (1937).

[11] L.D. Landau and E.M. Lifshitz, Statistical Physics (AddisonWesley, 1958) p. 353.

[12] R.K. Bhaduri, J. Dey, and M.A. Preston, Phys. Lett. 136B, 289 (1984).

[13] R.K. Bhaduri, R.S. Bhalerao, A. Khare, J. Law, and M.V.N. Murthy, Phys. Rev. Lett. 66, 523 (1991). In particular, see Eq. (16) and the subsequent discussion.

[14] J. Law, A. Suzuki, and R.K. Bhaduri, Phys. Rev.A 46, 4693 (1992).

[15] T.-L. Ho and Q. Zhao, Nature Physics 6, 131 (2010).

[16] X.-J. Liu, H. Hu, and P. Drummond, Phys. Rev. Lett. 102, 160401 (2009).

[17] D. Rakshit, K.M. Daily, and D. Blume, Phys. Rev A 85, 033634 (2012).

[18] R.K. Pathria, Statistical Mechanics (Pergammon Press, 1972) Appendix E, p. 505 